\title{
Lax Integrability and Soliton Solutions for a Nonisospectral Integrodifferential System
}

\author{
Sheng Zhang and Siyu Hong \\ School of Mathematics and Physics, Bohai University, Jinzhou 121013, China \\ Correspondence should be addressed to Sheng Zhang; szhangchina@126.com
}

Received 29 April 2017; Accepted 9 October 2017; Published 6 November 2017

Academic Editor: Pietro De Lellis

Copyright ( 2017 Sheng Zhang and Siyu Hong. This is an open access article distributed under the Creative Commons Attribution License, which permits unrestricted use, distribution, and reproduction in any medium, provided the original work is properly cited.

\begin{abstract}
Searching for integrable systems and constructing their exact solutions are of both theoretical and practical value. In this paper, Ablowitz-Kaup-Newell-Segur (AKNS) spectral problem and its time evolution equation are first generalized by embedding a new spectral parameter. Based on the generalized AKNS spectral problem and its time evolution equation, Lax integrability of a nonisospectral integrodifferential system is then verified. Furthermore, exact solutions of the nonisospectral integrodifferential system are formulated through the inverse scattering transform (IST) method. Finally, in the case of reflectionless potentials, the obtained exact solutions are reduced to $n$-soliton solutions. When $n=1$ and $n=2$, the characteristics of soliton dynamics of one-soliton solutions and two-soliton solutions are analyzed with the help of figures.
\end{abstract}

\section{Introduction}

Nonlinear phenomena involved in many fields such as physics, biology, chemistry, and mechanics are often related to nonlinear partial differential equations (PDEs). The investigation of exact solutions of nonlinear PDEs plays an important role because of its direct connection with dynamical processes in these nonlinear phenomena. Since the initialvalue problem of the Korteweg-de Vries (KdV) equation was exactly solved by the IST method [1], finding soliton solutions of nonlinear PDEs has become extremely active and some effective methods were proposed such as Hirota's bilinear method [2], Painlevé expansion [3], homogeneous balance method [4], and function expansion methods [5-10]. Among these methods, the IST [1] is a systematic method which has achieved considerable development and received a wide range of applications like those in [11-21] since it is put forward by Gardner, Greene, Kruskal, and Miura in 1967. One of the advantages of the IST is that it can solve a whole hierarchy of nonlinear PDEs associated with a certain spectral problem. As early as in 1976, the framework of IST with varying spectral parameter was introduced for the first time by Chen and Liu to the nonlinear Schrödinger (NLS) equation with a linear external potential [22] and by
Hirota and Satsuma to the KdV equation in nonuniform media [23]. Serkin et al. [24-28] pointed out that the soliton dynamics of nonautonomous ones which interact elastically and generally move with varying amplitudes, speeds, and spectra adapted both to the external potentials and to the dispersion and nonlinearity variations can be described in the framework of the IST theory with varying in time spectral parameter.

In soliton theory, nonlinear PDEs associated with some linear spectral problems can be generally classified as the isospectral equations which often describe solitary waves in lossless and uniform media and the nonisospectral equations describing the solitary waves in a certain type of nonuniform media. Specifically, when the spectral parameter of the associated linear spectral problem is independent of time, one could construct isospectral equations. While starting from the spectral problem with a time-dependent spectral parameter, nonisospectral equations are usually derived. In 1974, Ablowitz, Kaup, Newell, and Segur [21] successfully constructed a hierarchy of isospectral nonlinear PDEs; here it is written as

$$
\left(\begin{array}{l}
q \\
r
\end{array}\right)_{t}=L^{n}\left(\begin{array}{c}
-q \\
r
\end{array}\right), \quad(n=0,1,2, \ldots),
$$




$$
\begin{aligned}
L & =\sigma \partial+2\left(\begin{array}{c}
q \\
-r
\end{array}\right) \partial^{-1}(r, q), \\
\partial & =\frac{\partial}{\partial x}, \\
\partial^{-1} & =\frac{1}{2}\left(\int_{-\infty}^{x} \mathrm{~d} x-\int_{x}^{+\infty} \mathrm{d} x\right), \\
\sigma & =\left(\begin{array}{cc}
-1 & 0 \\
0 & 1
\end{array}\right),
\end{aligned}
$$

from the compatibility condition, that is, the zero curvature equation

$$
M_{t}-N_{x}+[M, N]=0
$$

of the following spectral problem

$$
\phi_{x}=M \phi, \quad M=\left(\begin{array}{cc}
-i k & q \\
r & i k
\end{array}\right), \phi=\left(\begin{array}{l}
\phi_{1} \\
\phi_{2}
\end{array}\right),
$$

and its evolution equation

$$
\phi_{t}=N \phi, \quad N=\left(\begin{array}{cc}
A & B \\
C & -A
\end{array}\right),
$$

where $q=q(x, t), r=r(x, t)$, and their derivatives of any order with respect to $x$ and $t$ are smooth functions which vanish as $x$ tends to infinity, the spectral parameter $k$ is independent with $x$ and $t$, and $A, B$, and $C$ are undetermined functions of $x, t, q, r$, and $k$.

When $n=2$, the isospectral AKNS hierarchy (1) gives

$$
\left(\begin{array}{l}
q \\
r
\end{array}\right)_{t}=\left(\begin{array}{l}
q_{x x x}-6 q r q_{x} \\
r_{x x x}-6 q r r_{x}
\end{array}\right)
$$

which includes the famous $\mathrm{KdV}$ equation $q_{t}=q_{x x x}+6 q q_{x}$ as a special case.

Subsequently, in the case of spectral parameter $k$ being dependent on time $t$, Calogero and Degasperis [29-31] and $\mathrm{Li}$ [32] proposed effective methods to derive different hierarchies of nonisospectral equations. For example, the nonisospectral AKNS hierarchy [20]

$$
\left(\begin{array}{l}
q \\
r
\end{array}\right)_{t}=L^{n}\left(\begin{array}{c}
-x q \\
x r
\end{array}\right), \quad(n=0,1,2, \ldots)
$$

can be derived from (3)-(5) equipped with $i k_{t}=(2 i k)^{n} / 2$. It is easy to see that when $n=0,1,2$, the nonisospectral AKNS hierarchy (1) gives the following nonisospectral systems:

$$
\begin{aligned}
& \left(\begin{array}{l}
q \\
r
\end{array}\right)_{t}=\left(\begin{array}{c}
-x q \\
x r
\end{array}\right), \\
& \left(\begin{array}{l}
q \\
r
\end{array}\right)_{t}=\left(\begin{array}{c}
q+x q_{x} \\
r+x r_{x}
\end{array}\right), \\
& \left(\begin{array}{l}
q \\
r
\end{array}\right)_{t}=\left(\begin{array}{c}
-2 q_{x}-x q_{x x}+2 q \partial^{-1} q r+2 x q^{2} r \\
2 r_{x}+x r_{x x}-2 r \partial^{-1} q r-2 x q^{2} r
\end{array}\right) .
\end{aligned}
$$

The aim of this paper is to generalize AKNS spectral problem (4) and its evolution equation (5) for testing the integrability of the following new and more general nonisospectral integrodifferential system:

$$
\begin{aligned}
& \left(\begin{array}{c}
q \\
r
\end{array}\right)_{t} \\
& =\left(\begin{array}{c}
-2 q_{x}-x q_{x x}+2 q \partial^{-1} q r+2 x q^{2} r+q+x q_{x}-x q-t q \\
2 r_{x}+x r_{x x}-2 r \partial^{-1} q r-2 x q^{2} r+r+x r_{x}+x r+t r
\end{array}\right)
\end{aligned}
$$

and extending the IST to system (9). With the help of (2), we can rewrite system (9) in the form

$$
\left(\begin{array}{l}
q \\
r
\end{array}\right)_{t}=L^{2}\left(\begin{array}{c}
-x q \\
x r
\end{array}\right)+L\left(\begin{array}{c}
-x q \\
x r
\end{array}\right)+\left(\begin{array}{c}
-x q \\
x r
\end{array}\right)+\left(\begin{array}{c}
-t q \\
t r
\end{array}\right),
$$

from which we can see that the nonisospectral integrodifferential system (9) with time-dependent coefficient terms is different from that in [33]

$$
\left(\begin{array}{l}
q \\
r
\end{array}\right)_{t}=L^{2 m+1}\left(\begin{array}{c}
-x q \\
x r
\end{array}\right)+L^{2 m}\left(\begin{array}{c}
-x q \\
x r
\end{array}\right)
$$

$$
(m=0,1,2, \ldots) .
$$

In order to construct system (9), in this paper we shall employ a new and more general spectral parameter $k$ which satisfies

$$
i k_{t}=\frac{1}{2} \sum_{n=0}^{2}(2 i k)^{n}
$$

It is easy to see that the nonisospectral parameter $\eta_{t}=(2 \eta)^{n} / 2$ in [33] is a special case of (12). Here $i k$ in (12) is equivalent to $\eta$ in [33]. On the other hand, we shall generalize the matrix $N$ in [33]

$$
\left.N\right|_{(q, r)=(0,0)}=\left(\begin{array}{cc}
-\frac{1}{2}(2 i k) x & 0 \\
0 & \frac{1}{2}(2 i k) x
\end{array}\right)
$$

to the following form:

$$
\begin{aligned}
& \left.N\right|_{(q, r)=(0,0)} \\
& \quad\left(\begin{array}{cc}
-\frac{1}{2}\left[\sum_{n=0}^{2}(2 i k)^{n}\right] x-\frac{1}{2} & 0 \\
0 & \frac{1}{2}\left[\sum_{n=0}^{2}(2 i k)^{n}\right] x+\frac{1}{2}
\end{array}\right) .
\end{aligned}
$$

In the very recent work [34], we let the parameter $k$ satisfy

$$
i k_{t}=\frac{1}{2} \sin 2 i k=\sum_{j=0}^{+\infty}(-1)^{j} \frac{1}{(2 j+1) !}(2 i k)^{2 j+1}
$$

and employed 


$$
\left.N\right|_{(q, r)=(0,0)}\left(\begin{array}{cc}
-\frac{1}{2} x \sin 2 i k & 0 \\
0 & \frac{1}{2} x \sin 2 i k
\end{array}\right) \text {, }
$$

then a general nonisospectral integrodifferential system of the form

$$
\left(\begin{array}{l}
q \\
r
\end{array}\right)_{t}=\sum_{j=0}^{+\infty}(-1)^{j} \frac{1}{(2 j+1) !} !^{2 j+1}\left(\begin{array}{c}
-x q \\
x r
\end{array}\right)
$$

is constructed. Equation (17) can be rewritten as

$$
\begin{aligned}
\left(\begin{array}{l}
q \\
r
\end{array}\right)_{t}= & L\left(\begin{array}{c}
-x q \\
x r
\end{array}\right)-\frac{1}{3 !} L^{3}\left(\begin{array}{c}
-x q \\
x r
\end{array}\right)+\frac{1}{5 !} L^{5}\left(\begin{array}{c}
-x q \\
x r
\end{array}\right) \\
& +\sum_{j=3}^{+\infty}(-1)^{j} \frac{1}{(2 j+1) !} L^{2 j+1}\left(\begin{array}{c}
-x q \\
x r
\end{array}\right)
\end{aligned}
$$

which has the expansion in part

$$
\begin{aligned}
\left(\begin{array}{l}
q \\
r
\end{array}\right)_{t}= & \left(\begin{array}{l}
q+x q_{x}-\frac{1}{2} q_{x x}-\frac{1}{6} x q_{x x x}+\frac{1}{3} q_{x} \partial^{-1}(q r)+\frac{2}{3} x q r q_{x}+\frac{1}{3} x q^{2} r_{x}+\frac{4}{3} q \partial^{-1}\left(q_{x} r\right)+\frac{1}{3} x q r q_{x x}-\frac{1}{3} x q^{2} r_{x x} \\
r+x r_{x}-\frac{1}{2} r_{x x}-\frac{1}{6} x r_{x x x}+\frac{1}{3} r_{x} \partial^{-1}(q r)+\frac{2}{3} x q r r_{x}+\frac{1}{3} x r^{2} q_{x}+\frac{4}{3} r \partial^{-1}\left(q r_{x}\right)+\frac{1}{3} x q r r_{x x}-\frac{1}{3} x r^{2} q_{x x}
\end{array}\right) \\
& +\sum_{j=2}^{+\infty}(-1)^{j} \frac{1}{(2 j+1) !} L^{2 j+1}\left(\begin{array}{c}
-x q \\
x r
\end{array}\right) .
\end{aligned}
$$

Obviously, there is substantial difference between system (9) and (17) in [34]. It is because, except for the term

$$
L\left(\begin{array}{c}
-x q \\
x r
\end{array}\right)=\left(\begin{array}{c}
q+x q_{x} \\
r+x r_{x}
\end{array}\right)
$$

the other three terms of (10)

$$
\begin{aligned}
& L^{2}\left(\begin{array}{c}
-x q \\
x r
\end{array}\right)=\left(\begin{array}{c}
-2 q_{x}-x q_{x x}+2 q \partial^{-1} q r+2 x q^{2} r \\
2 r_{x}+x r_{x x}-2 r \partial^{-1} q r-2 x q^{2} r
\end{array}\right), \\
&\left(\begin{array}{c}
-x q \\
x r
\end{array}\right),\left(\begin{array}{c}
-t q \\
t r
\end{array}\right)
\end{aligned}
$$

cannot be contained in (18). Due to appearance of the third term of (21), system (9) is a variable-coefficient system with not only space-dependent coefficients but also timedependent coefficients. However, (17) has not such a term with time-dependent coefficients. In fact, the different selections for (12) and (14) lead to the difference between system (9) and (17).

The rest of the paper is organized as follows. In Section 2, we prove the Lax integrability of system (9) by generalizing AKNS spectral problem (4) and its evolution equation (5). In Section 3, system (9) is solved via the IST. As a result, the uniform formulae of exact solutions are obtained. In the special case of reflectionless potentials, the obtained exact solutions are reduced to $n$-soliton solutions. In Section 4, we conclude this paper.

\section{Lax Integrability}

Theorem 1. Suppose that the function A in (5) has the form

$$
A=\partial^{-1}(r, q)\left(\begin{array}{c}
-B \\
C
\end{array}\right)-\frac{1}{2}\left[\sum_{n=0}^{2}(2 i k)^{n}\right] x-\frac{1}{2},
$$

then the nonisospectral integrodifferential system (9) can be derived from (3) and thus system (9) is Lax integrable.

Proof. Firstly, by virtue of (3) equipped with the new spectral parameter $k$ satisfying (12) we have

$$
\begin{aligned}
A_{x} & =q C-r B-\frac{1}{2} \sum_{n=0}^{2}(2 i k)^{n}, \\
q_{t} & =B_{x}+2 i k B+2 q A, \\
r_{t} & =C_{x}-2 i k C-2 r A .
\end{aligned}
$$

Supposing that

$$
A=\partial^{-1}(r, q)\left(\begin{array}{c}
-B \\
C
\end{array}\right)-\frac{1}{2}\left[\sum_{n=0}^{2}(2 i k)^{n}\right] x-\frac{1}{2}
$$

from (24) and (25), we have

$$
\begin{aligned}
\left(\begin{array}{l}
q \\
r
\end{array}\right)_{t}= & L\left(\begin{array}{c}
-B \\
C
\end{array}\right)-2 i k\left(\begin{array}{c}
-B \\
C
\end{array}\right)+\sum_{n=0}^{2}(2 i k)^{n}\left(\begin{array}{c}
-x q \\
x r
\end{array}\right) \\
& +\left(\begin{array}{c}
-t q \\
t r
\end{array}\right),
\end{aligned}
$$

by the use of (2).

We next suppose that

$$
\left(\begin{array}{c}
-B \\
C
\end{array}\right)=\sum_{l=1}^{2}\left(\begin{array}{c}
-b_{l} \\
c_{l}
\end{array}\right)(2 i k)^{2-l}
$$


and substitute (28) into (27). Then comparing the coefficients of $2 i k$ in (27) yields

$$
\begin{aligned}
(2 i k)^{0}:\left(\begin{array}{c}
q \\
r
\end{array}\right)_{t} & =L\left(\begin{array}{c}
-b_{2} \\
c_{2}
\end{array}\right)+\left(\begin{array}{c}
-x q \\
x r
\end{array}\right)+\left(\begin{array}{c}
-t q \\
t r
\end{array}\right), \\
(2 i k)^{1}:\left(\begin{array}{c}
-b_{2} \\
c_{2}
\end{array}\right) & =L\left(\begin{array}{c}
-b_{1} \\
c_{1}
\end{array}\right)+\left(\begin{array}{c}
-x q \\
x r
\end{array}\right), \\
(2 i k)^{2}:\left(\begin{array}{c}
-b_{1} \\
c_{1}
\end{array}\right) & =\left(\begin{array}{c}
-x q \\
x r
\end{array}\right),
\end{aligned}
$$

from which we derive (10). Finally, the substitution of (2) into (10), we arrive at the nonisospectral integrodifferential system (9). Thus, the proof is completed.

\section{Soliton Solutions}

In this section, we first determine the time dependence of scattering data for the AKNS spectral problem (4) with the generalized time evolution equation (5) caused by (22). Based on the determined scattering data, we then construct exact solutions of nonisospectral integrodifferential system (9). We finally reduce the obtained exact solutions to soliton solutions and analyze the soliton dynamics.

\subsection{The Time Dependence of Scattering Data}

Theorem 2. The scattering data $\kappa_{j}(t), \bar{\kappa}_{m}(t), c_{j}(t), \bar{c}_{m}(t)$, $R(t, k)=\beta(t, k) / \alpha(t, k)$, and $\bar{R}(t, k)=\bar{\beta}(t, k) / \bar{\alpha}(t, k)(j=1$, $2, \ldots, n ; m=1,2, \ldots, \bar{n})$ for the generalized spectral problem (4) possess the following time dependence:

$$
\begin{aligned}
\kappa_{j t}(t) & =-\frac{i}{2} \sum_{m=0}^{2}\left[2 i \kappa_{j}(t)\right]^{m}, \\
c_{j}(t) & =c_{j}(0) e^{\int_{0}^{t}\left[1+2 i \kappa_{j}(s)\right] \mathrm{d} s}, \\
\alpha(t, k) & =\alpha(0, k), \\
\beta(t, k) & =\beta(0, k), \\
\bar{\kappa}_{m t}(t) & =-\frac{i}{2} \sum_{l=0}^{2}\left[2 i \bar{\kappa}_{j}(t)\right]^{l}, \\
\bar{c}_{m}(t) & =\bar{c}_{m}(0) e^{-\int_{0}^{t}\left[1+2 i \bar{\kappa}_{m}(s)\right] \mathrm{d} s}, \\
\bar{\alpha}(t, k) & =\bar{\alpha}(0, k), \\
\bar{\beta}(t, k) & =\bar{\beta}(0, k),
\end{aligned}
$$

where $c_{j}(0), \bar{c}_{m}(0), R(0, k)=\beta(0, k) / \alpha(0, k)$, and $\bar{R}(0, k)=$ $\bar{\beta}(0, k) / \bar{\alpha}(0, k)$ are the scattering data of the generalized spectral problem (4) in the case of $(q(0, x), r(0, x))^{T}$.

Proof. It is easy to see that if $\phi(x, k)$ is a solution of the generalized spectral problem (4) then $P(x, k)=\phi_{t}(x, k)-$
$N \phi(x, k)$ is also a solution of generalized spectral problem (4). Therefore, $P(x, k)$ can be represented by $\phi(x, k)$ and $\widetilde{\phi}(x, k)$ which also satisfies the generalized spectral problem (4) but is independent of $\phi(x, k)$; that is, there exist two functions $\gamma(t, k)$ and $\tau(t, k)$ such that

$$
\begin{aligned}
\phi_{t}(x, k)-N \phi(x, k)= & \gamma(t, k) \phi(x, k) \\
& +\tau(t, k) \widetilde{\phi}(x, k) .
\end{aligned}
$$

Firstly, we consider the discrete spectral $k=\kappa_{j}\left(\operatorname{Im} \kappa_{j}>\right.$ $0)$. Since $\phi\left(x, \kappa_{j}\right)$ decays exponentially while $\widetilde{\phi}(x, k)$ must increase exponentially as $x \rightarrow+\infty$, we then have $\tau(t, k)=0$. Thus, (31) is simplified as

$$
\phi_{t}\left(x, \kappa_{j}\right)-N \phi\left(x, \kappa_{j}\right)=\gamma\left(t, \kappa_{j}\right) \phi\left(x, \kappa_{j}\right) .
$$

Left-multiplying (32) by the inner product $\left(\phi_{2}\left(x, \kappa_{j}\right), \phi_{1}(x\right.$, $\left.\kappa_{j}\right)$ ) yields

$$
\begin{aligned}
& \frac{\mathrm{d}}{\mathrm{d} t} \phi_{1}\left(x, \kappa_{j}\right) \phi_{2}\left(x, \kappa_{j}\right)-\left[C \phi_{1}^{2}\left(x, \kappa_{j}\right)+B \phi_{2}^{2}\left(x, \kappa_{j}\right)\right] \\
& \quad=2 \gamma\left(t, \kappa_{j}\right) \phi_{1}\left(x, \kappa_{j}\right) \phi_{2}\left(x, \kappa_{j}\right) .
\end{aligned}
$$

Presuming $\phi\left(x, \kappa_{j}\right)$ to be the normalization eigenfunction and noting that $2 \int_{-\infty}^{\infty} c_{j}^{2} \phi_{1}\left(x, \kappa_{j}\right) \phi_{2}\left(x, \kappa_{j}\right) \mathrm{d} x=1$, we have

$$
\gamma\left(t, \kappa_{j}\right)=-c_{j}^{2} \int_{-\infty}^{\infty}\left[C \phi_{1}^{2}\left(x, \kappa_{j}\right)+B \phi_{2}^{2}\left(x, \kappa_{j}\right)\right] \mathrm{d} x .
$$

For convenience, we rewrite (34) as

$$
\gamma\left(t, \kappa_{j}\right)=-c_{j}^{2}\left(\left(\phi_{2}^{2}\left(x, \kappa_{j}\right), \phi_{1}^{2}\left(x, \kappa_{j}\right)\right)^{T},(B, C)^{T}\right),
$$

where the following inner product had been used

$$
\begin{aligned}
& (f(x), g(x)) \\
& \quad=\int_{-\infty}^{\infty}\left(f_{1}(x) g_{1}(x)+f_{2}(x) g_{2}(x)\right) \mathrm{d} x
\end{aligned}
$$

for arbitrary two vectors $f(x)=\left(f_{1}(x), f_{2}(x)\right)^{T}$ and $g(x)=$ $\left(g_{1}(x), g_{2}(x)\right)^{T}$.

Using (4), we have

$$
\begin{aligned}
& \phi_{1 x}\left(x, \kappa_{j}\right)+i \kappa_{j} \phi_{1}\left(x, \kappa_{j}\right)=q(x) \phi_{2}\left(x, \kappa_{j}\right), \\
& \phi_{2 x}\left(x, \kappa_{j}\right)-i \kappa_{j} \phi_{2}\left(x, \kappa_{j}\right)=r(x) \phi_{1}\left(x, \kappa_{j}\right)
\end{aligned}
$$

and hence obtain

$$
\begin{aligned}
& {\left[\phi_{1}\left(x, \kappa_{j}\right) \phi_{2}\left(x, \kappa_{j}\right)\right]_{x}} \\
& \quad=q(x) \phi_{2}^{2}\left(x, \kappa_{j}\right)+r(x) \phi_{1}^{2}\left(x, \kappa_{j}\right) .
\end{aligned}
$$

Integrating (38) with respect to $x$ from $-\infty$ to $+\infty$ yields

$$
\begin{gathered}
\int_{-\infty}^{\infty}\left[q(x) \phi_{2}^{2}\left(x, \kappa_{j}\right)+r(x) \phi_{1}^{2}\left(x, \kappa_{j}\right)\right] \mathrm{d} x \\
=\int_{-\infty}^{\infty}\left[\phi_{1}\left(x, \kappa_{j}\right) \phi_{2}\left(x, \kappa_{j}\right)\right]_{x} \mathrm{~d} x=0 .
\end{gathered}
$$


On the other hand, we rewrite (28) as

$$
\begin{aligned}
\left(\begin{array}{l}
B \\
C
\end{array}\right) & =\sum_{l=1}^{2} \sum_{s=1}^{l} \bar{L}^{s-1}\left(\begin{array}{l}
x q \\
x r
\end{array}\right)(2 i k)^{2-l}, \\
\bar{L} & =\sigma \partial-2\left(\begin{array}{l}
q \\
r
\end{array}\right) \partial^{-1}(-r, q)
\end{aligned}
$$

and then obtain

$$
\begin{aligned}
& \gamma\left(t, \kappa_{j}\right)=-c_{j}^{2}\left(\left(\phi_{2}^{2}\left(x, \kappa_{j}\right), \phi_{1}^{2}\left(x, \kappa_{j}\right)\right)^{T},(B, C)^{T}\right) \\
& =-c_{j}^{2}\left(\left(\phi_{2}^{2}\left(x, \kappa_{j}\right), \phi_{1}^{2}\left(x, \kappa_{j}\right)\right)^{T}, \sum_{l=1}^{2} \sum_{s=1}^{l} \bar{L}^{s-1}\left(\begin{array}{l}
x q \\
x r
\end{array}\right)\right. \\
& \left.\cdot(2 i k)^{2-l}\right)=-c_{j}^{2} \sum_{l=1}^{2} \sum_{s=1}^{l}\left(2 i \kappa_{j}\right)^{2-l} \\
& \cdot\left(\left(\phi_{2}^{2}\left(x, \kappa_{j}\right), \phi_{1}^{2}\left(x, \kappa_{j}\right)\right)^{T}, \bar{L}^{l-1}\left(\begin{array}{l}
q \\
r
\end{array}\right)\right)=\frac{1}{2} \\
& \cdot \sum_{s=1}^{2} s\left(2 i \kappa_{j}\right)^{s-1} .
\end{aligned}
$$

Then (32) becomes

$$
\begin{aligned}
\phi_{t} & \left(x, \kappa_{j}\right)-N \phi\left(x, \kappa_{j}\right) \\
= & \frac{1}{2}\left[\sum_{s=1}^{2} s\left(2 i \kappa_{j}\right)^{s-1}\right] \phi\left(x, \kappa_{j}\right) .
\end{aligned}
$$

Noting that

$N$

$$
\begin{gathered}
\longrightarrow\left(\begin{array}{cc}
-\frac{1}{2}\left[\sum_{n=0}^{2}\left(2 i \kappa_{j}\right)^{n}\right] x-\frac{1}{2} & 0 \\
0 & \frac{1}{2}\left[\sum_{n=0}^{2}\left(2 i \kappa_{j}\right)^{n}\right] x+\frac{1}{2}
\end{array}\right), \\
\phi\left(x, \kappa_{j}\right) \longrightarrow c_{j}\left(\begin{array}{l}
0 \\
1
\end{array}\right) e^{i \kappa_{j} x}, \\
\phi_{t}\left(x, \kappa_{j}\right) \rightarrow c_{j t}\left(\begin{array}{l}
0 \\
1
\end{array}\right) e^{i \kappa_{j} x}+x c_{j} \kappa_{j t}\left(\begin{array}{l}
0 \\
1
\end{array}\right) e^{i \kappa_{j} x},
\end{gathered}
$$

as $x \rightarrow+\infty$, then from (42)-(43) we have

$$
\begin{aligned}
& \kappa_{j t}=-\frac{i}{2} \sum_{n=0}^{2}\left(2 i \kappa_{j}\right)^{n}, \\
& c_{j t}=c_{j}\left[\frac{1}{2} \sum_{s=1}^{2} s\left(2 i \kappa_{j}\right)^{s-1}+\frac{1}{2}\right] .
\end{aligned}
$$

In a similar way, we obtain

$$
\begin{aligned}
& \bar{\kappa}_{j t}=-\frac{i}{2} \sum_{n=0}^{2}\left(2 i \bar{\kappa}_{j}\right)^{n}, \\
& \bar{c}_{j t}=-\bar{c}_{j}\left[\frac{1}{2} \sum_{s=1}^{2} s\left(2 i \bar{\kappa}_{j}\right)^{s-1}+\frac{1}{2}\right] .
\end{aligned}
$$

Secondly, we consider $k$ as a real continuous spectral and take a solution $\varphi(x, k)$ of the generalized spectral problem (4), then the solution of the generalized spectral problem (4)

$$
P_{1}(x, k)=\varphi_{t}(x, k)-N \varphi(x, k)
$$

can be represented linearly by $\varphi(x, k)$ and $\bar{\varphi}(x, k)$ which also satisfies the generalized spectral problem (4) but is independent of $\varphi(x, k)$, that is, there exist two functions $\omega(t, k)$ and $\vartheta(t, k)$ such that

$$
\begin{aligned}
\varphi_{t}(x, k)-N \varphi(x, k)= & \omega(t, k) \varphi(x, k) \\
& +\vartheta(t, k) \bar{\varphi}(x, k) .
\end{aligned}
$$

Using the asymptotical properties

$$
\begin{aligned}
& \varphi_{t}(x, k) \longrightarrow-i k_{t} x\left(\begin{array}{l}
1 \\
0
\end{array}\right) e^{-i k x}, \\
& \varphi(x, k) \longrightarrow\left(\begin{array}{l}
1 \\
0
\end{array}\right) e^{-i k x}, \\
& \bar{\varphi}(x, k) \longrightarrow\left(\begin{array}{c}
0 \\
-1
\end{array}\right) e^{i k x},
\end{aligned}
$$

as $x \rightarrow-\infty$, from (47) we obtain

$$
\begin{aligned}
& \vartheta(t, k)=0, \\
& \omega(t, k)=0 .
\end{aligned}
$$

Substituting the Jost relationship $\varphi(x, k)=\alpha(t, k) \bar{\phi}(x, k)+$ $\beta(t, k) \phi(x, k)$ into (47) yields

$$
\begin{aligned}
& {[\alpha(t, k) \bar{\phi}(x, k)+\beta(t, k) \phi(x, k)]_{t}} \\
& \quad-N[\alpha(t, k) \bar{\phi}(x, k)+\beta(t, k) \phi(x, k)]=0 .
\end{aligned}
$$

Letting $x \rightarrow-\infty$ and using

$$
\begin{aligned}
& \phi(x, k) \longrightarrow\left(\begin{array}{l}
0 \\
1
\end{array}\right) e^{i k x}, \\
& \bar{\phi}(x, k) \longrightarrow\left(\begin{array}{l}
1 \\
0
\end{array}\right) e^{-i k x},
\end{aligned}
$$

from (50) we derive

$$
\begin{aligned}
& \frac{\mathrm{d} \alpha(t, k)}{\mathrm{d} t}=0, \\
& \frac{\mathrm{d} \beta(t, k)}{\mathrm{d} t}=0 .
\end{aligned}
$$


Similarly, we have

$$
\begin{aligned}
& \frac{\mathrm{d} \bar{\alpha}(t, k)}{\mathrm{d} t}=0, \\
& \frac{\mathrm{d} \bar{\beta}(t, k)}{\mathrm{d} t}=0 .
\end{aligned}
$$

Finally, solving (44), (45), (52), and (53) yields (30). We therefore finish the proof.

3.2. Exact Solutions and Soliton Solutions. According to Theorem 1 and the results in [20], we have the following Theorem 3.

Theorem 3. Given the scattering data for the generalized spectral problem (4), the nonisospectral integrodifferential system (9) has exact solutions as follows:

$$
\begin{aligned}
& q(x, t)=-2 K_{1}(t, x, x), \\
& r(x, t)=\frac{K_{2 x}(t, x, x)}{K_{1}(t, x, x)},
\end{aligned}
$$

where $K(t, x, y)=\left(K_{1}(t, x, y), K_{2}(t, x, y)\right)^{T}$ satisfies the Gel'fand-Levitan-Marchenko (GLM) integral equation:

$$
\begin{aligned}
& K(t, x, y)-\left(\begin{array}{l}
0 \\
1
\end{array}\right) \bar{F}(t, x+y) \\
& \quad+\left(\begin{array}{l}
0 \\
1
\end{array}\right) \int_{x}^{\infty} F(t, z+x) \bar{F}(t, z+y) \mathrm{d} z \\
& \quad+\int_{x}^{\infty} K(t, x, s) \int_{x}^{\infty} F(t, z+s) \bar{F}(t, z+y) \mathrm{d} z \mathrm{~d} s \\
& =0,
\end{aligned}
$$

with

$$
\begin{aligned}
& F(t, x)=\frac{1}{2 \pi} \int_{-\infty}^{\infty} R(t, k) e^{i k x} \mathrm{~d} k+\sum_{j=1}^{n} c_{j}^{2} e^{i \kappa_{j} x}, \\
& \bar{F}(t, x)=\frac{1}{2 \pi} \int_{-\infty}^{\infty} \bar{R}(t, k) e^{-i k x} \mathrm{~d} k-\sum_{j=1}^{\bar{n}} \bar{c}_{j}^{2} e^{-i \bar{\kappa}_{j} x} .
\end{aligned}
$$

In order to give explicit form of solutions (54), we consider $R(t, k)=\bar{R}(t, k)=0$. In this reflectionless potentials case, the GLM integral equation (55) can be solved exactly. For convenience, we use $K(t, x, y)=\left(K_{1}(t, x, y), K_{2}(t\right.$, $x, y))^{T}$ to rewrite $(55)$ as

$$
\begin{gathered}
K_{1}(t, x, y)-\bar{F}_{d}(t, x+y)+\int_{x}^{\infty} K_{1}(t, x, s) \\
\cdot \int_{x}^{\infty} F_{d}(t, z+s) \bar{F}_{d}(t, z+y) \mathrm{d} z \mathrm{~d} s=0, \\
K_{2}(t, x, y)+\int_{x}^{\infty} F_{d}(t, z+x) \bar{F}_{d}(t, z+y) \mathrm{d} z \\
+\int_{x}^{\infty} K_{2}(t, x, s) \\
\cdot \int_{x}^{\infty} F_{d}(t, z+s) \bar{F}_{d}(t, z+y) \mathrm{d} z \mathrm{~d} s=0 .
\end{gathered}
$$

Using (56), we can get

$$
\begin{aligned}
& \int_{x}^{\infty} F_{d}(t, s+z) \bar{F}_{d}(t, z+y) \mathrm{d} z \\
& =-\sum_{j=1}^{n} \sum_{m=1}^{\bar{n}} \frac{i c_{j}^{2}(t) \bar{c}_{m}^{2}(t)}{\kappa_{j}-\bar{\kappa}_{m}} e^{i \kappa_{j}(x+s)-i \bar{\kappa}_{m}(x+y)} .
\end{aligned}
$$

Supposing that

$$
\begin{aligned}
& K_{1}(x, y, t)=\sum_{p=1}^{\bar{n}} \bar{c}_{p}(t) g_{p}(t, x) e^{-i \bar{\kappa}_{p} y}, \\
& K_{2}(x, y, t)=\sum_{p=1}^{\bar{n}} \bar{c}_{p}(t) h_{p}(t, x) e^{-i \bar{\kappa}_{p} y}
\end{aligned}
$$

and substituting (59) into (57) yield

$$
\begin{aligned}
& g_{m}(t, x)+\bar{c}_{m}(t) e^{-i \bar{\kappa}_{m} x} \\
& +\sum_{j=1}^{n} \sum_{p=1}^{\bar{n}} \frac{c_{j}^{2}(t) \bar{c}_{m}(t) \bar{c}_{p}(t)}{\left(\kappa_{j}-\bar{\kappa}_{m}\right)\left(\kappa_{j}-\bar{\kappa}_{p}\right)} e^{i\left(2 \kappa_{j}-\bar{\kappa}_{m}-\bar{\kappa}_{p}\right) x} g_{p}(x, t) \\
& =0, \\
& h_{m}(x, t)-\sum_{j=1}^{n} \frac{1}{\left(\kappa_{j}-\bar{\kappa}_{m}\right)} c_{j}^{2}(t) \bar{c}_{m}(t) e^{i\left(2 \kappa_{j}-\bar{\kappa}_{m}\right) x} \\
& +\sum_{j=1}^{n} \sum_{p=1}^{\bar{n}} \frac{c_{j}^{2}(t) \bar{c}_{m}(t) \bar{c}_{p}(t)}{\left(\kappa_{j}-\bar{\kappa}_{m}\right)\left(\kappa_{j}-\bar{\kappa}_{p}\right)} e^{i\left(2 \kappa_{j}-\bar{\kappa}_{m}-\bar{\kappa}_{p}\right) x} h_{p}(x, t) \\
& =0, \quad(m=1,2, \ldots, \bar{n}) . \\
& \text { Introducing the vectors } \\
& g(t, x)=\left(g_{1}(t, x), g_{2}(t, x), \ldots, g_{\bar{n}}(t, x)\right)^{T}, \\
& h(t, x)=\left(h_{1}(t, x), h_{2}(t, x), \ldots, h_{\bar{n}}(t, x)\right)^{T}, \\
& \Lambda=\left(c_{1}(t) e^{i \kappa_{1} x}, c_{2}(t) e^{i \kappa_{2} x}, \ldots, c_{n}(t) e^{i \kappa_{n} x}\right)^{T}, \\
& \bar{\Lambda}=\left(\bar{c}_{1}(t) e^{-i \bar{\kappa}_{1} x}, \bar{c}_{2}(t) e^{-i \bar{\kappa}_{2} x}, \ldots, \bar{c}_{n}(t) e^{-i \bar{\kappa}_{n} x}\right)^{T},
\end{aligned}
$$


we can write (60) in the matrix forms

$$
\begin{aligned}
& W(t, x) g(t, x)=-\bar{\Lambda}(t, x), \\
& W(t, x) h(t, x)=i P(t, x) \Lambda(t, x),
\end{aligned}
$$

where

$$
\begin{aligned}
W(t, x) & =E+P(t, x) P^{T}(t, x), \\
P(t, x) & =\left(\frac{c_{j}(t) \bar{c}_{m}(t)}{\kappa_{j}-\bar{\kappa}_{m}} e^{i\left(\kappa_{j}-\bar{\kappa}_{m}\right) x}\right)_{\bar{n} \times n},
\end{aligned}
$$

and $E$ is a $\bar{n} \times \bar{n}$ unit matrix.

Supposing $W^{-1}(t, x)$ exists, then we have

$$
\begin{aligned}
& g(t, x)=-W^{-1}(t, x) \bar{\Lambda}(t, x), \\
& h(t, x)=i W^{-1}(t, x) P(t, x) \Lambda(t, x) .
\end{aligned}
$$

Substituting (64) into (59) we have

$$
\begin{aligned}
& K_{1}(x, y, t)=-\operatorname{tr}\left(W^{-1}(t, x) \bar{\Lambda}(t, x) \bar{\Lambda}^{T}(t, y)\right), \\
& K_{2}(x, y, t) \\
& \quad=i \operatorname{tr}\left(W^{-1}(t, x) E(t, x) \Lambda(t, x) \bar{\Lambda}^{T}(t, y)\right),
\end{aligned}
$$

where $\operatorname{tr}(\cdot)$ means the trace of a given matrix.

Substituting (65) into (54), we obtain the following $n$ soliton solutions of the nonisospectral integrodifferential system (9):

$$
\begin{aligned}
& q(x, t)=2 \operatorname{tr}\left(W^{-1}(t, x) \bar{\Lambda}(t, x) \bar{\Lambda}^{T}(t, x)\right), \\
& r(x, t) \\
& \quad=-\frac{(\mathrm{d} / \mathrm{d} x) \operatorname{tr}\left(W^{-1}(t, x) E(t, x)(\mathrm{d} / \mathrm{d} x) E^{T}(t, x)\right)}{\operatorname{tr}\left(W^{-1}(t, x) \bar{\Lambda}(t, x) \bar{\Lambda}^{T}(t, x)\right)} .
\end{aligned}
$$

Particularly, when $n=\bar{n}=1$, (66) give the one-soliton solutions:

9

$$
\begin{aligned}
& =\frac{2 \bar{c}_{1}^{2}(0) e^{-2 i \bar{\kappa}_{1} x-2 \int_{0}^{t}\left[1+2 i \bar{\kappa}_{1}(s)\right] \mathrm{d} s}}{1+\left(c_{1}^{2}(0) \bar{c}_{1}^{2}(0) /\left(\kappa_{1}-\bar{\kappa}_{1}\right)^{2}\right) e^{2 i\left(\kappa_{1}-\bar{\kappa}_{1}\right) x+4 i \int_{0}^{t}\left[\kappa_{1}(s)-\bar{\kappa}_{1}(s)\right] \mathrm{d} s}}, \\
& r \\
& =\frac{2 c_{1}^{2}(0) e^{2 i \kappa_{1} x+2 \int_{0}^{t}\left[1+2 i \kappa_{1}(s)\right] \mathrm{d} s}}{1+\left(c_{1}^{2}(0) \bar{c}_{1}^{2}(0) /\left(\kappa_{1}-\bar{\kappa}_{1}\right)^{2}\right) e^{2 i\left(\kappa_{1}-\bar{\kappa}_{1}\right) x+4 i \int_{0}^{t}\left[\kappa_{1}(s)-\bar{\kappa}_{1}(s)\right] \mathrm{d} s}},
\end{aligned}
$$

where $\kappa_{1}$ and $\bar{\kappa}_{1}$ are determined by the Riccati equations

$$
\begin{gathered}
\kappa_{1 t}=-\frac{i}{2} \sum_{n=0}^{2}\left(2 i \kappa_{1}\right)^{n}, \\
\bar{\kappa}_{1 t}=-\frac{i}{2} \sum_{n=0}^{2}\left(2 i \bar{\kappa}_{1}\right)^{n} .
\end{gathered}
$$

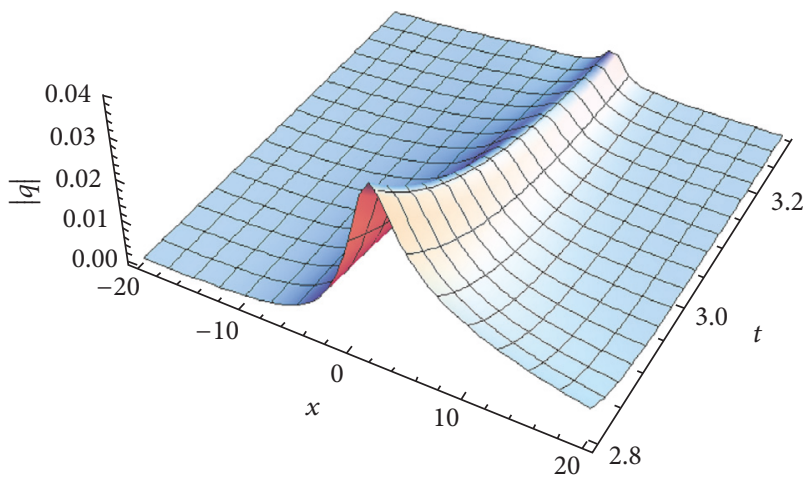

FIGURE 1: Local spatial structure of one-soliton solution (67) with $\kappa_{1}$ and $\bar{\kappa}_{1}$ satisfying (69).

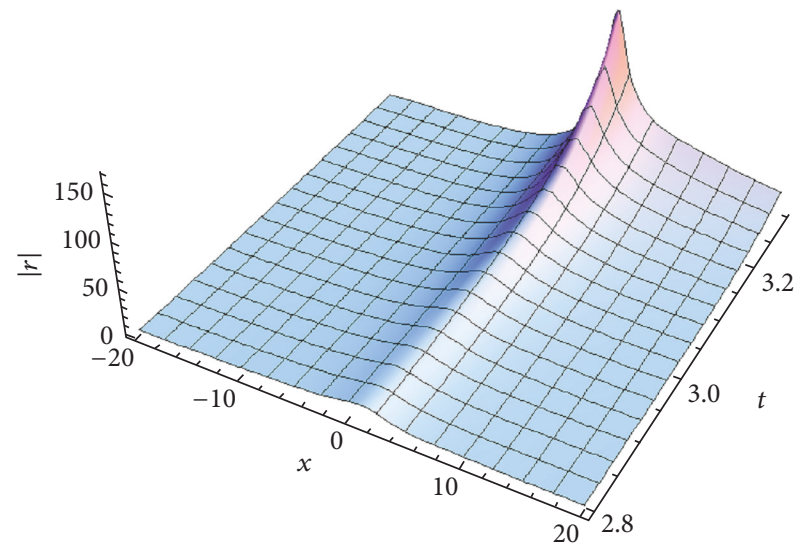

FIGURE 2: Local spatial structure of one-soliton solution (68).

3.3. Soliton Dynamics. In this part, we further investigate the soliton dynamics of system (9) by means of one-soliton solutions and two-soliton solutions. To determine $\kappa_{1}$ and $\bar{\kappa}_{1}$ of (69), we employ Zhang et al's direct algorithm [35] of expfunction method and gain two special solutions of (69):

$$
\begin{aligned}
\kappa_{1} & =\frac{i}{4}(1+i \sqrt{3}) \\
& +\frac{\sqrt{3}}{2\left[1+\left(\left(i+\sqrt{3}-4 \kappa_{1}(0)\right) /\left(-i+\sqrt{3}+4 \kappa_{1}(0)\right)\right) e^{i \sqrt{3} t}\right]}, \\
\bar{\kappa}_{1} & =\frac{i}{4}(1+i \sqrt{3}) \\
& +\frac{\sqrt{3}}{2\left[1+\left(\left(i+\sqrt{3}-4 \bar{\kappa}_{1}(0)\right) /\left(-i+\sqrt{3}+4 \bar{\kappa}_{1}(0)\right)\right) e^{i \sqrt{3} t}\right]} .
\end{aligned}
$$

In Figures 1 and 2, two local spatial structures of one-soliton solutions (67) and (68) are shown by selecting the parameters as $\kappa_{1}(0)=1, \bar{\kappa}_{1}(0)=-0.5, c_{1}(0)=1$, and $\bar{c}_{1}(0)=$ 0.2 . We can see from Figures 1 and 2 that the local spatial structures of one-soliton solutions (67) and (68) possess the bell-shaped characteristics. The dynamical evolutions of twosoliton solutions determined by (66) are shown in Figures 3 and 4 , where the parameters are selected as $\kappa_{1}(0)=1$, $\bar{\kappa}_{1}(0)=0.5, \kappa_{2}(0)=-0.3, \bar{\kappa}_{2}(0)=-1.5, c_{1}(0)=-0.01$, 


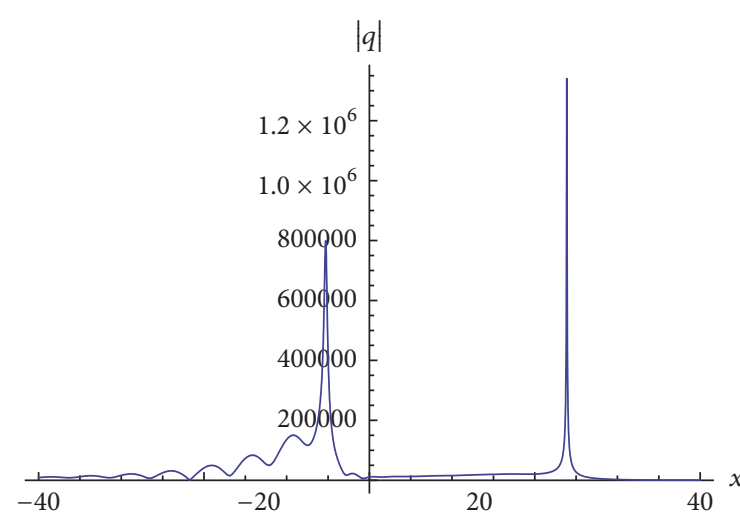

(a)

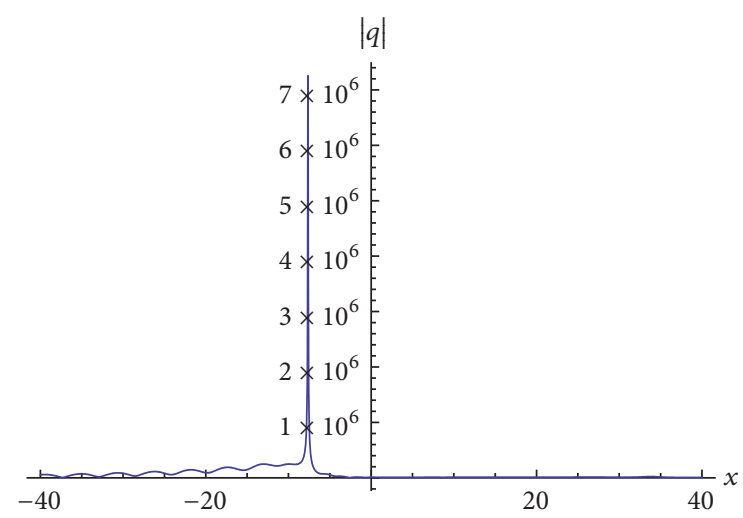

(c)

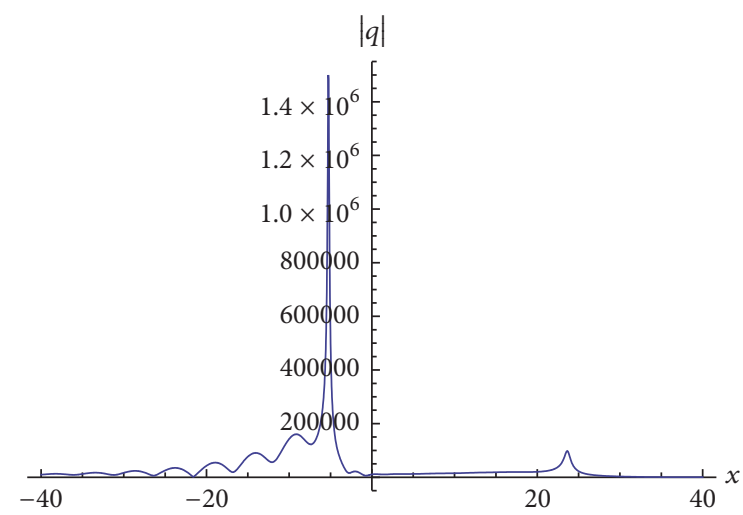

(b)

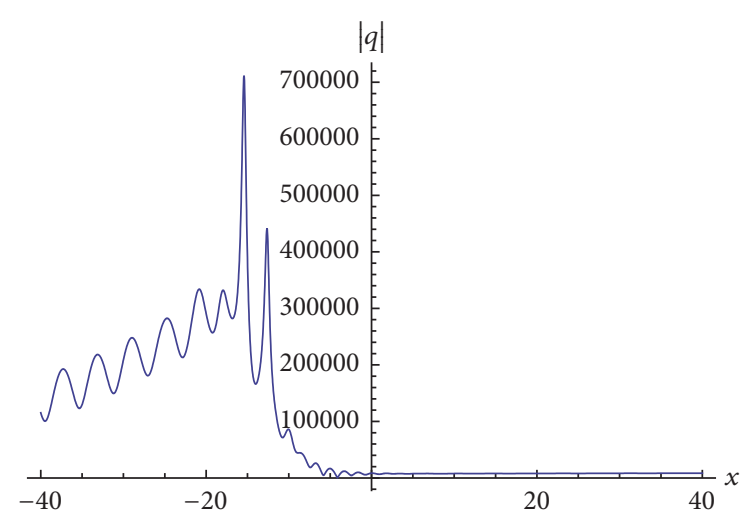

(d)

Figure 3: Dynamical evolutions of two-soliton solution determined by (66) at different times: (a) $t=-11.112$, (b) $t=-11.104$, (c) $t=-11$, and (d) $t=-10.94$.

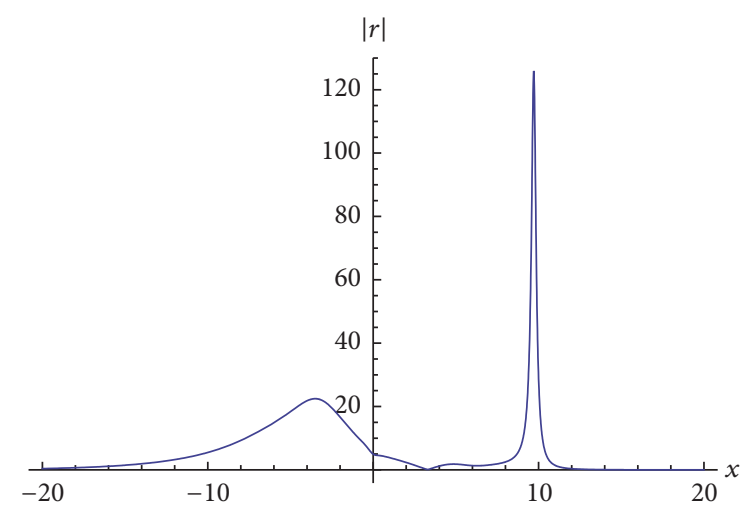

(a)

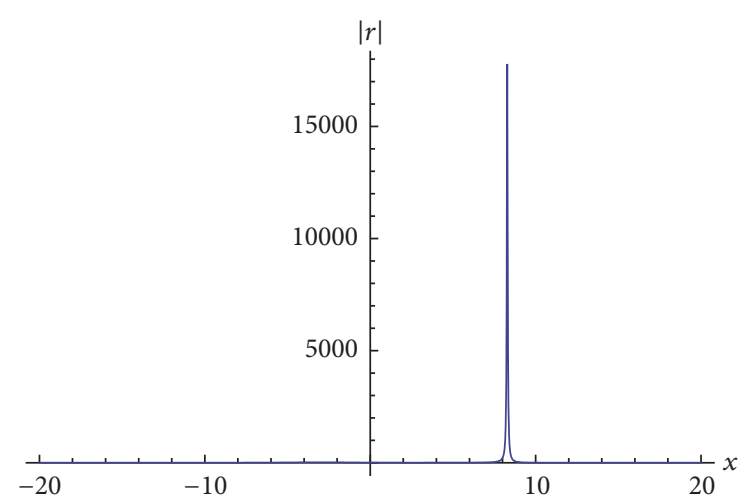

(c)

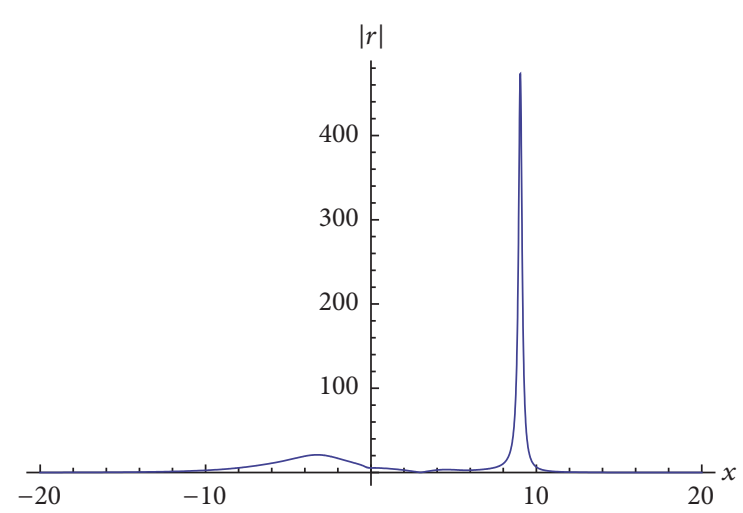

(b)

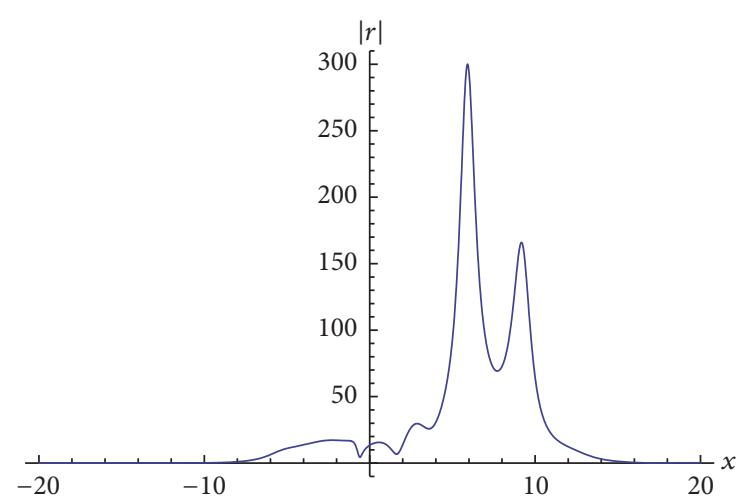

(d)

Figure 4: Dynamical evolutions of two-soliton solution determined by (66) at different times: (a) $t=2.8,(\mathrm{~b}) t=2.9$, (c) $t=3$, and (d) $t=3.3$. 
$\bar{c}_{1}(0)=0.25, c_{2}(0)=0.5$, and $\bar{c}_{2}(0)=-0.1$, respectively. Figures 3 and 4 show that the inelastic scatterings can happen between two-soliton solutions determined by (66).

\section{Conclusions and Discussions}

In summary, we have verified Lax integrability of the new and more general nonisospectral integrodifferential system (9). This is due to the generalizations on AKNS spectral problem (4) and its time evolution equation (5) by embedding a new spectral parameter. To exactly solve the nonisospectral integrodifferential system (9), the IST is employed. As a result, exact solutions (54) are obtained. In the case of reflectionless potentials, the obtained exact solutions (54) are reduced to $n$-soliton solutions (66). When $n=1$ and $n=2$, the characteristics of soliton dynamics of one-soliton solutions and two-soliton solutions are analyzed with the help of figures. To the best of our knowledge, the nonisospectral integrodifferential system (9), the exact solutions (54), and the $n$-soliton solutions (66) have not been reported in literatures. How to construct other nonisospectral integrodifferential systems and their soliton solutions in the framework of IST method is worthy of study. This is our task in the future.

\section{Conflicts of Interest}

The authors declare that there are no conflicts of interest regarding the publication of this article.

\section{Acknowledgments}

This work was supported by the Natural Science Foundation of China (11547005), the Natural Science Foundation of Liaoning Province of China (201705040007), the Natural Science Foundation of Education Department of Liaoning Province of China (LZ2017002), and the Liaoning BaiQianWan Talents Program of Liaoning Province of China (2013921055).

\section{References}

[1] C. S. Gardner, J. M. Greene, M. D. Kruskal, and R. M. Miura, "Method for solving the Korteweg-deVries equation," Physical Review Letters, vol. 19, no. 19, pp. 1095-1097, 1967.

[2] R. Hirota, "Exact solution of the korteweg-de vries equation for multiple Collisions of solitons," Physical Review Letters, vol. 27, no. 18, pp. 1192-1194, 1971.

[3] J. Weiss, M. Tabor, and G. Carnevale, “The Painlevé property for partial differential equations," Journal of Mathematical Physics, vol. 24, no. 3, pp. 522-526, 1983.

[4] M. Wang, "Exact solutions for a compound KdV-Burgers equation," Physics Letters A, vol. 213, no. 5-6, pp. 279-287, 1996.

[5] E. Fan, "Travelling wave solutions in terms of special functions for nonlinear coupled evolution systems," Physics Letters A, vol. 300, no. 2-3, pp. 243-249, 2002.

[6] J.-H. He and X.-H. Wu, "Exp-function method for nonlinear wave equations," Chaos, Solitons \& Fractals, vol. 30, no. 3, pp. 700-708, 2006.
[7] S. Zhang and T.-C. Xia, "A generalized auxiliary equation method and its application to $(2+1)$-dimensional asymmetric Nizhnik-Novikov-Vesselov equations," Journal of Physics A: Mathematical and General, vol. 40, no. 2, pp. 227-248, 2007.

[8] S. Zhang and D.-D. Liu, "The third kind of Darboux transformation and multisoliton solutions for generalized Broer-Kaup equations," Turkish Journal of Physics, vol. 39, no. 2, pp. 165-177, 2015.

[9] C.-Q. Dai and Y.-Y. Wang, "Controllable combined Peregrine soliton and Kuznetsov-Ma soliton in PT-symmetric nonlinear couplers with gain and loss," Nonlinear Dynamics, vol. 80, no. 1-2, pp. 715-721, 2015.

[10] R. Abazari, "Biswas, Solitary wave solutions of coupled Boussinesq equation," Complixity, vol. 21, no. S2, pp. 151-155, 2016, http://onlinelibrary.wiley.com/advanced/search.

[11] A. I. Nachman and M. J. Ablowitz, "A multidimensional inversescattering method," Studies in Applied Mathematics, vol. 71, no. 3, pp. 243-250, 1984.

[12] W. L. Chan and K.-S. Li, "Nonpropagating solitons of the variable coefficient and nonisospectral Korteweg-de Vries equation," Journal of Mathematical Physics, vol. 30, no. 11, pp. 25212526, 1989.

[13] B. Z. Xu and S. Q. Zhao, "Inverse scattering transformation for the variable coefficient sine-Gordon type equation," Applied Mathematics-A Journal of Chinese Universities Series B, vol. 9, no. 4, pp. 331-337, 1994.

[14] G. Biondini and G. Kovačič, "Inverse scattering transform for the focusing nonlinear Schrödinger equation with nonzero boundary conditions," Journal of Mathematical Physics, vol. 55, 2014.

[15] S. Zhang and X.-D. Gao, "Mixed spectral AKNS hierarchy from linear isospectral problem and its exact solutions," Open Physics, vol. 13, no. 1, pp. 310-322, 2015.

[16] S. Zhang, B. Xu, and H.-Q. Zhang, "Exact solutions of a KdV equation hierarchy with variable coefficients," International Journal of Computer Mathematics, vol. 91, no. 7, pp. 1601-1616, 2014.

[17] S. Zhang and D. Wang, "Variable-coefficient nonisospectral Toda lattice hierarchy and its exact solutions," PramanaJournal of Physics, vol. 85, no. 6, pp. 1143-1156, 2015.

[18] S. Zhang and X. Gao, "Exact solutions and dynamics of a generalized AKNS equations associated with the nonisospectral depending on exponential function," Journal of Nonlinear Sciences and Applications, vol. 9, no. 6, pp. 4529-4541, 2016.

[19] X. D. Gao and S. Zhang, "Time-dependent-coefficient AKNS hierarchy and its exact multi-soliton solutions," International Journal of Applied Science and Mathematics, vol. 3, no. 2, pp. 7275, 2016.

[20] D. Y. Chen, Introduction of Soliton, Science Press, Beijing, 2006.

[21] M. J. Ablowitz and P. A. Clarkson, Solitons, nonlinear evolution equations and inverse scattering, Cambridge University Press, Cambridge, 1991.

[22] H. H. Chen and C. S. Liu, "Solitons in nonuniform media," Physical Review Letters, vol. 37, no. 11, pp. 693-697, 1976.

[23] R. Hirota and J. Satsuma, "N-Soliton Solution of the K-dV Equation with Loss and Nonuniformity Terms," Journal of the Physical Society of Japan, vol. 41, no. 6, pp. 2141-2142, 1976.

[24] V. N. Serkin and A. Hasegawa, "Novel soliton solutions of the nonlinear Schrodinger equation model," Physical Review Letters, vol. 85, no. 21, pp. 4502-4505, 2000. 
[25] V. N. Serkin and T. L. Belyaeva, "Optimal control of optical soliton parameters: Part 1 . The Lax representation in the problem of soliton management," Quantum Electronics, vol. 31, no. 11, pp. 1007-1015, 2001.

[26] V. N. Serkin, A. Hasegawa, and T. L. Belyaeva, "Nonautonomous solitons in external potentials," Physical Review Letters, vol. 98, 2007.

[27] V. N. Serkin, A. Hasegawa, and T. L. Belyaeva, "Solitary waves in nonautonomous nonlinear and dispersive systems: Nonautonomous solitons," Journal of Modern Optics, vol. 57, no. 14-15, pp. 1456-1472, 2010.

[28] V. N. Serkin, A. Hasegawa, and T. L. Belyaeva, "Nonautonomous matter-wave solitons near the Feshbach resonance," Physical Review, vol. 81, 2010.

[29] F. Calogero and A. Degasperis, "Extension of the spectral transform method for solving nonlinear evolution equations," Lettere Al Nuovo Cimento, vol. 22, no. 4, pp. 131-137, 1978.

[30] F. Calogero and A. Degasperis, "Extension of the spectral transform method for solving nonlinear evolution equations.II," Lettere Al Nuovo Cimento, vol. 22, no. 7, pp. 263-269, 1978.

[31] F. Calogero and A. Degasperis, "Exact solution via the spectral transform of a generalization with linearly $\mathrm{x}$-dependent coefficients of the modified Korteweg-de-Vires equation," Lettere Al Nuovo Cimento, vol. 22, no. 7, pp. 270-273, 1978.

[32] Y. S. Li, "A class of evolution equations and the spectral deformation," Science in China Series A: Mathematics, vol. 25, no. 9, pp. 911-917, 1982.

[33] T.-k. Ning, D.-y. Chen, and D.-j. Zhang, "The exact solutions for the nonisospectral AKNS hierarchy through the inverse scattering transform," Physica A, vol. 339, no. 3-4, pp. 248-266, 2004.

[34] S. Zhang and J. H. Li, "On nonisospectral AKNS system with infinite number of terms and its exact solutions," IAENG International Journal of Applied Mathematics, vol. 47, no. 1, pp. 89-96, 2017.

[35] S. Zhang, J. H. Li, and L. Y. Zhang, "A direct algorithm of expfunction method for non-linear evolution equations in fluids," Thermal Science, vol. 20, no. 3, pp. 881-884, 2016. 


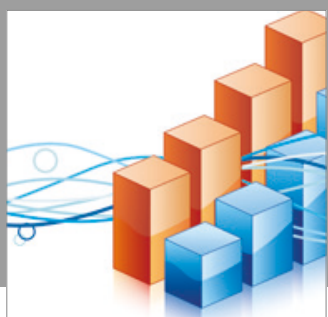

Advances in

Operations Research

vatersals

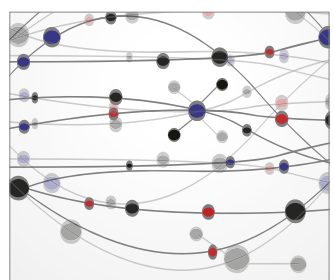

\section{The Scientific} World Journal
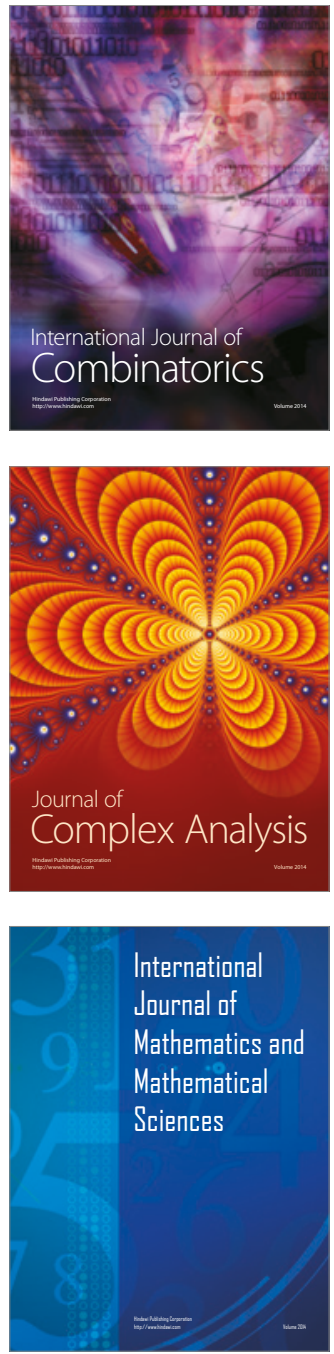
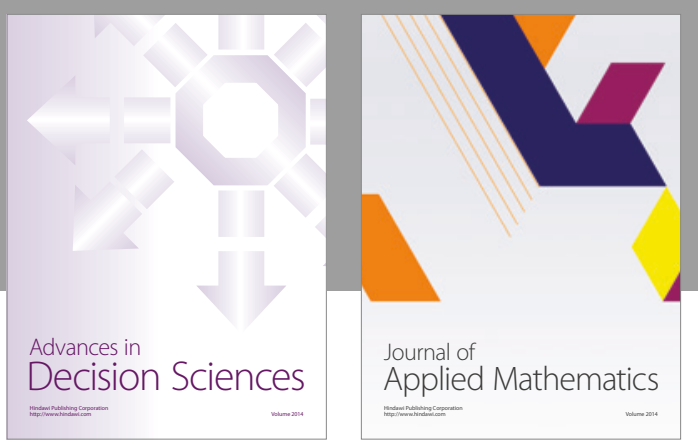

Algebra

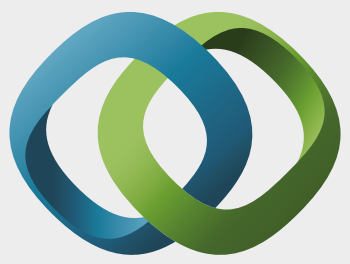

\section{Hindawi}

Submit your manuscripts at

https://www.hindawi.com
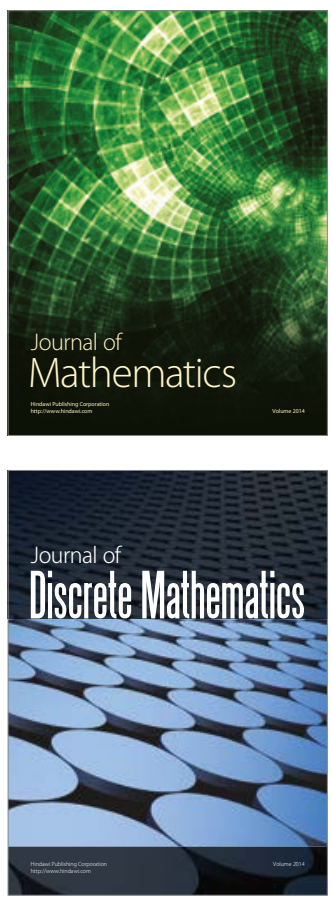

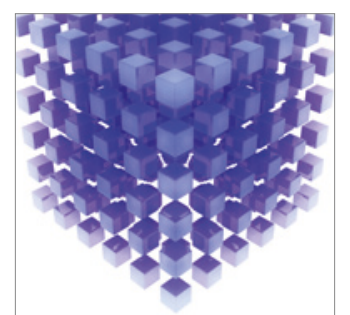

Mathematical Problems in Engineering
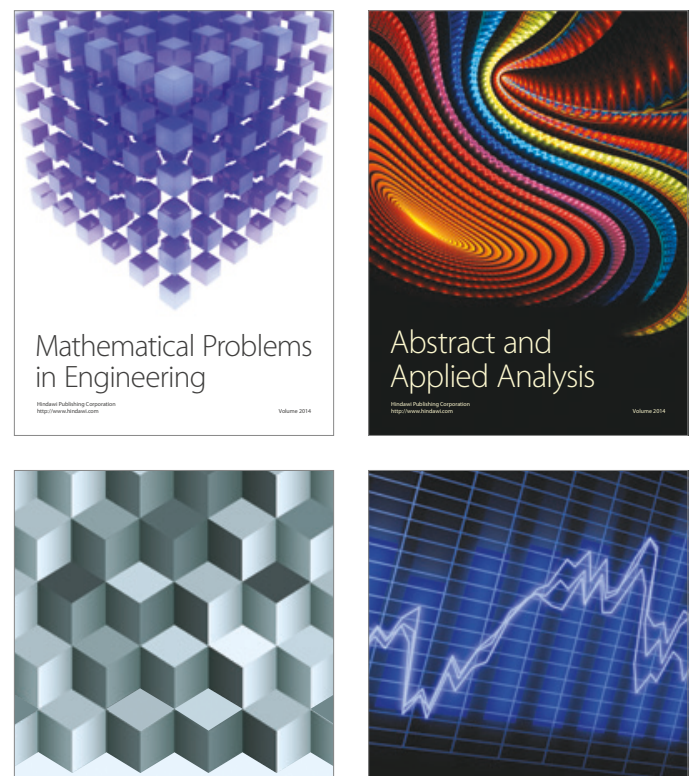

Journal of

Function Spaces

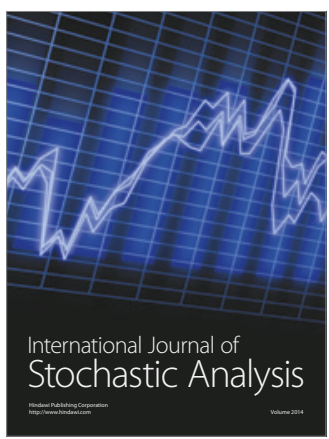

Probability and Statistics
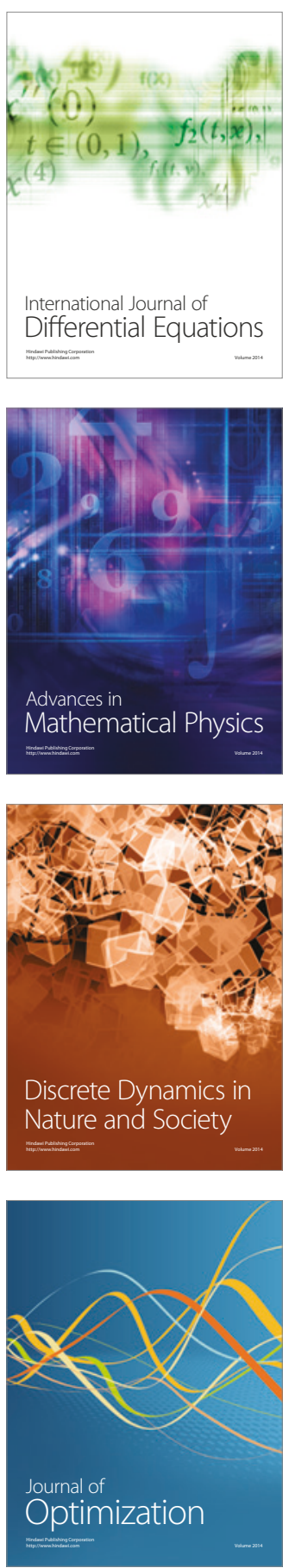